\title{
Shock compression of precompressed deuterium
}

M. R. Armstrong, J. C. Crowhurst, J. M. Zaug, S. Bastea, A. F. Goncharov, B. Militzer

August 3, 2011

Shock compression of precompressed deuterium Chicago, IL, United States

June 26, 2011 through July 2, 2011 
This document was prepared as an account of work sponsored by an agency of the United States government. Neither the United States government nor Lawrence Livermore National Security, LLC, nor any of their employees makes any warranty, expressed or implied, or assumes any legal liability or responsibility for the accuracy, completeness, or usefulness of any information, apparatus, product, or process disclosed, or represents that its use would not infringe privately owned rights. Reference herein to any specific commercial product, process, or service by trade name, trademark, manufacturer, or otherwise does not necessarily constitute or imply its endorsement, recommendation, or favoring by the United States government or Lawrence Livermore National Security, LLC. The views and opinions of authors expressed herein do not necessarily state or reflect those of the United States government or Lawrence Livermore National Security, LLC, and shall not be used for advertising or product endorsement purposes. 


\title{
Shock compression of precompressed deuterium
}

\author{
M. R. Armstrong ${ }^{1}$, J. C. Crowhurst ${ }^{1}$, A. F. Goncharov ${ }^{2}$, J. M. Zaug ${ }^{1}$, S. Bastea ${ }^{1}$, and B. \\ Militzer $^{3}$ \\ ${ }^{I}$ Chemical Sciences Division, Lawrence Livermore National Laboratory, Livermore CA 94551 \\ ${ }^{2}$ Geophysical Laboratory, Carnegie Institute of Washington, Washington, DC 20015 \\ ${ }^{3}$ Astronomy department, UC Berkeley, Berkley, CA 94720
}

\begin{abstract}
Here we report quasi-isentropic dynamic compression and thermodynamic characterization of solid, precompressed deuterium over an ultrafast time scale $(<100 \mathrm{ps})$ and a microscopic length scale $(<1 \mu \mathrm{m})$. We further report a fast transition in shock wave compressed solid deuterium that is consistent with the ramp to shock transition, with a time scale of less than $10 \mathrm{ps}$. These results suggest that high-density dynamic compression of hydrogen may be possible on microscopic length scales.
\end{abstract}

Keywords: Hydrogen, deuterium, dynamic compression, ramp compression, static compression, shock wave.

PACS: 46.40.Cd, 62.20.-x.

\section{INTRODUCTION}

Cold, dense hydrogen ${ }^{1}$ exhibits a number of exotic phenomena [1,2], and the thermodynamic properties of hydrogen at very high density are essential to a diverse range of topics including the fundamental physics of solids, the physics of giant planets, and inertial confinement fusion. Dynamic compression can generate much higher pressures and temperatures than other methods, but fast ${ }^{2}$ compression of hydrogen cannot obtain high density [3].

States with higher density and lower temperature may be obtained via two methods,

${ }^{1}$ Although the experiments described here are for deuterium (which gives better shock impedance matching than hydrogen), under the thermodynamic conditions addressed in this work analogous results for hydrogen are easily derived from measurements in deuterium.

${ }^{2}$ Here, "fast" corresponds to a compression time scale comparable to or faster than the steady shock wave rise time, and "slow" corresponds to a compression time scale much slower than the thermodynamic equilibration time. both which have been intensively developed recently. First, static precompression may be used to increase the initial density of the material (see refs. [3] and [4]). Second, the material may be compressed more slowly than the steady state shock rise time, i.e. ramp, or quasi-isentropic, compression. Substantially less heat is deposited under ramp compression compared to shock compression to the same pressure, enabling generation of lower temperature, higher density thermodynamic states for a given final pressure.

\section{EXPERIMENTS}

We apply ultrafast compression methods $[4,5]$ to dynamically compress solid deuterium from initial static pressures above $20 \mathrm{GPa}$ at room temperature to final pressures as high as $44 \mathrm{GPa}$, over a time scale of $10 \mathrm{~s}$ of picoseconds, using the method of ref. [4], as shown in Fig. 1.

Dynamic compression is achieved via laser ablation of a $\sim 1 \mu \mathrm{m}$ thick Al layer on the culet of one diamond in a conventional diamond anvil cell 


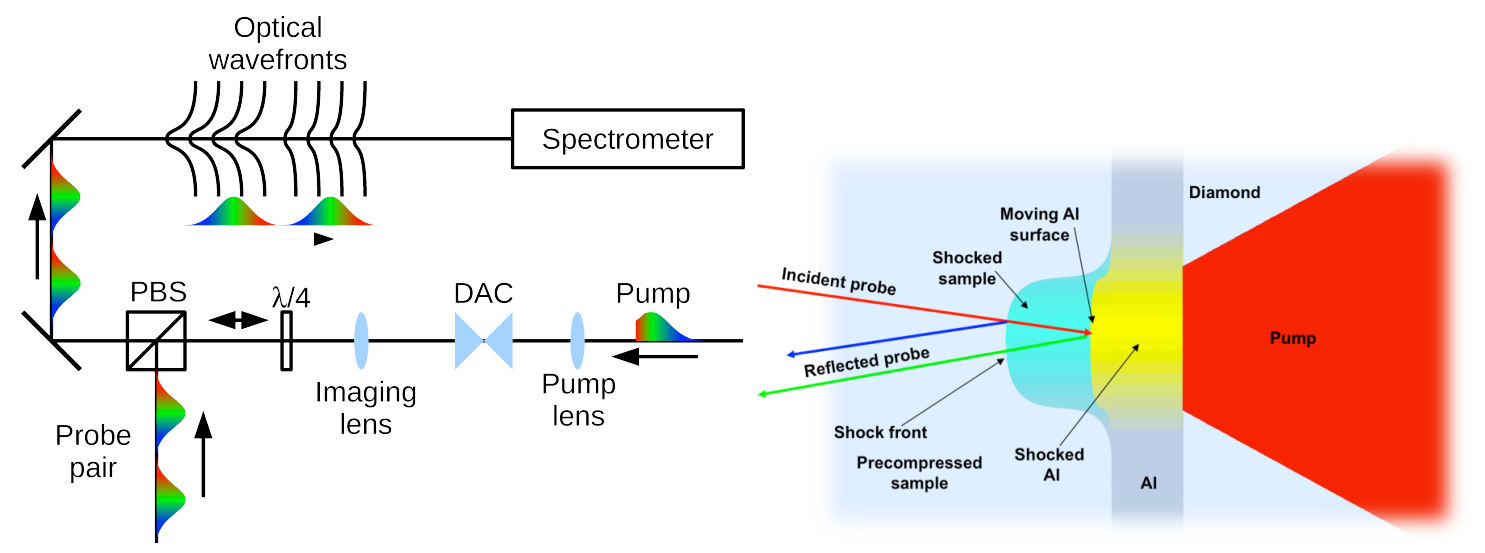

Figure 1: (Left) The optical setup, PBS is a polarizing beam splitter. (Right) A close-up of the shocked region in the DAC. Probe light reflects from the shock front and the ablator/sample interface. These two reflections are Doppler shifted to different frequencies and beat, producing a signal from which shock parameters may be derived.

which has been loaded with deuterium. Energy deposition in the ablator drives a shock wave through the Al layer into the sample.

Simultaneously, a pair of broadband $(\sim 25 \mathrm{~nm}$ FWHM bandwidth) chirped optical pulses near 800 $\mathrm{nm}$ wavelength are used as an interferometric probe of the shocked state in the deuterium, as described in ref. [4]. This diagnostic is essentially ultrafast VISAR. For expansion of a shocked free surface, this method measures an optical phase shift over a ps-scale time delay, providing a measurement of surface velocity in direct analogy to VISAR measurements, but with picosecond time resolution. The time resolution of this method can be as high as 3 ps for a 300 ps FWHM observation window with a $25 \mathrm{~nm}$ FWHM bandwidth pulse. For the data shown in this work, a moving average with a $5 \mathrm{ps}$ width is applied to all data, reducing the

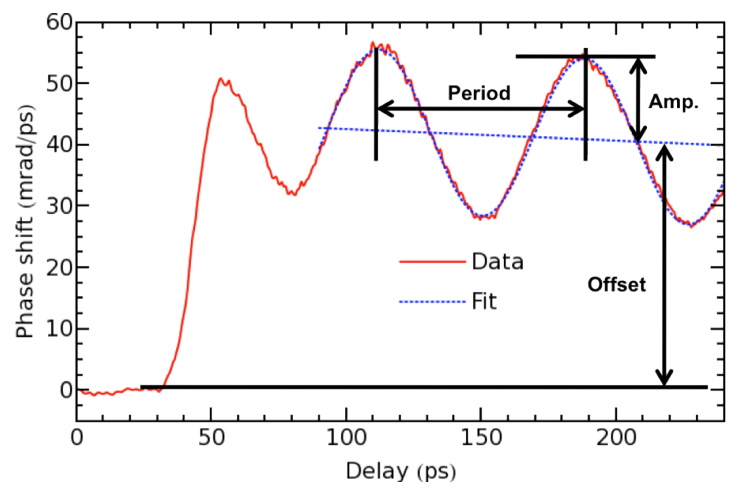

Figure 2: The typical form of the ultrafast VISAR signal from a shocked sample, in this case, shocked water. time resolution to $\sim 5 \mathrm{ps}$.

For steady shock waves, this method obtains data with a characteristic form shown in Fig. 2. From this data, three parameters are estimated, the average phase offset, the amplitude of the sinusoidal oscillation, and the period of the oscillation. From these measured parameters, the shock velocity, particle velocity and index of refraction behind the shock front may be estimated.

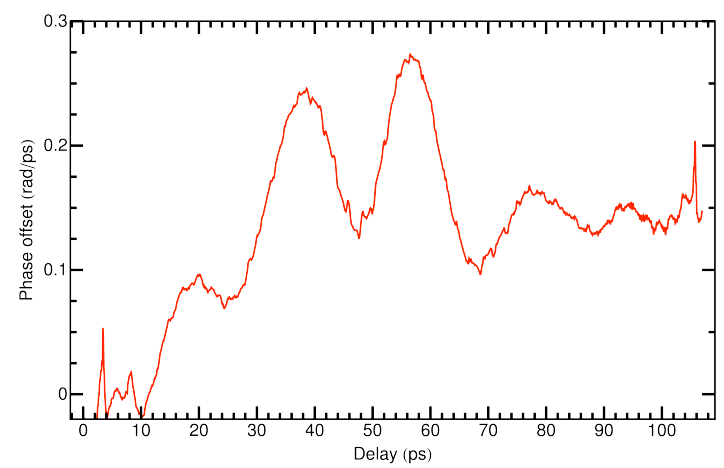

Figure 3: Ultrafast VISAR data from deuterium at an initial pressure of $22 \mathrm{GPa}$.

In contrast, analogous data from precompressed deuterium gives a qualitatively different signal, as shown in Fig. 3. In particular, the oscillation amplitude and offset do not reach steady state values on a time scale less than the period of oscillation, and there is a rapid transition in the data at around $65 \mathrm{ps}$. Fits to several datasets with similar characteristics are shown in Fig. 4. All fits were placed in time just previous to the fast transition and fit over a range of about 1.5 cycles of 
the oscillation, where the wave offset and amplitude were steady.

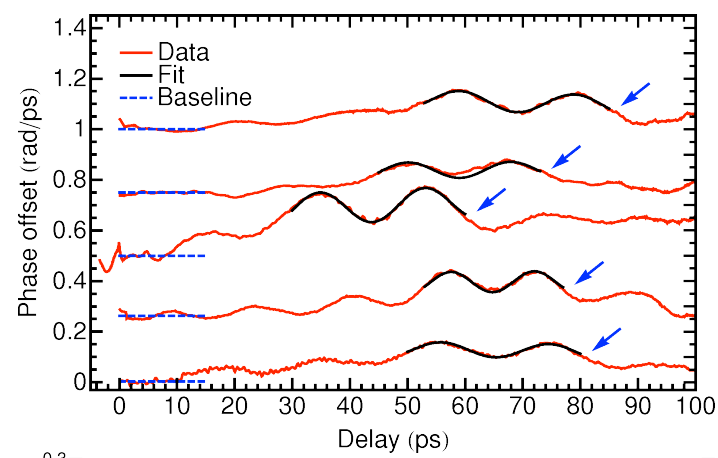

Figure 4: Several qualitatively similar datasets from deuterium shocked from initial pressures ranging from 22-24 GPa. Arrows point to where transitions occur in the data.

Velocities given by the fit and analysis are shown in Fig. 5, along with calculations of the Hugoniot of deuterium from $24 \mathrm{GPa}$ pressure, and the calculated sound speed plus particle speed vs. particle speed for the deuterium isentrope starting from $24 \mathrm{GPa}$. Although generally the measured wave speeds (at a given particle velocity) are faster than the isentrope, they are more consistent in magnitude and slope with the calculated wave speeds along the isentrope.

Further, the fast transitions in these data occur at times consistent with simulations of ramp steepening given a $20-40$ ps rise time in the compression wave from the $\mathrm{Al}$ ablator, consistent with measurements of the $\mathrm{Al}$ rise time outside the DAC. These observations suggest that the ramp to shock transition occurs within $\sim 100$ ps of the wave

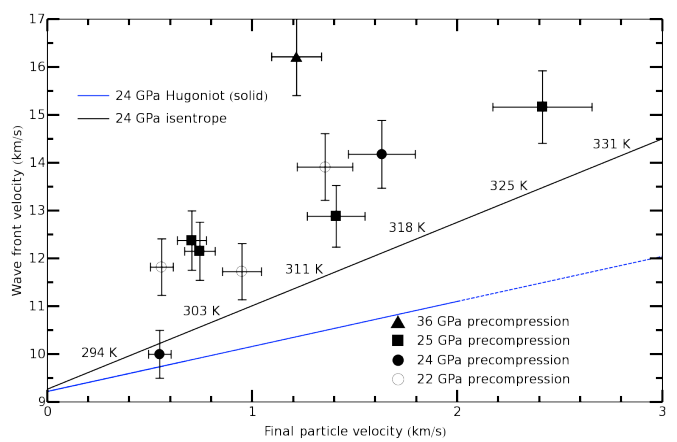

Figure 5: Estimated wave and particle velocities from time domain ultrafast VISAR data in deuterium arrival at the $\mathrm{Al} /$ deuterium interface. These data further suggest that the ramp to shock transition interferes with the coherent reflection of the probe from the wave front, which in turn eliminates sinusoidal oscillation in the data. This hypothesis is qualitatively consistent with theoretical speculation regarding the nature of the ramp to shock transition [6], where the convergence of acoustic characteristics in a steepening ramp results in acoustic instabilities propagating out from the point of convergence.

We note two caveats with respect to the analysis. First, estimates of the particle velocity depend on the amplitude of the oscillation in the time domain data (via the reflectivity of the wave front). For shock waves, the wave front is effectively a Fresnel interface, but ramp waves (even on this time scale) may not act as a step interface in the index of refraction. This results in an underestimate of the particle velocity (but very little change in the estimate of the wave velocity), so the particle velocity estimates shown in Fig. 5 are probably too low. Based on simulations of the ultrafast VISAR signal for comparable compression, we estimate these particle velocity estimates to be no more than $30 \%$ lower than the actual particle velocity, and more accurate estimates may be possible with reasonable assumptions about the material compression. Second, the measured wave speed is not the peak wave speed, but an aggregate wave speed whose value depends on the strain rate distribution in the compression wave. For a symmetric compression wave, the measured wave speed is the average of the sound speed in the uncompressed material and the peak wave speed. Taken together, these errors in the velocity do not substantially impact the primary conclusion of this work, that deuterium can be isentropically compressed on an ultrafast time scale.

\section{CONCLUSION}

Using ultrafast interferometry to characterize an ultrafast compression wave in deuterium at pressures of greater than $20 \mathrm{GPa}$, we observe a signal that is consistent with a steepening ramp (quasi-isentropic) compression wave for propagation time less than $100 \mathrm{ps}$, followed by a rapid $(<10 \mathrm{ps})$ qualitative change in the signal. The 
timing of this rapid change in the signal is consistent with ramp wave compression steepening into a shock wave, which destroys the optical coherence of the wave, providing a signature of the ramp to shock transition in ultrafast VISAR data.

\section{ACKNOWLEDGEMENTS}

Thanks to Larry Fried, Evan Reed, Chris Grant, Libby Glascoe, and Vitali Prakapenka. This work was supported by LLNL LDRD project 05-ERD039, the US Department of Energy, Office of Basic Energy Sciences via EFREE (H. K. Mao, PI) as part of an Energy Frontier Research Center, and with partial funding from the DTRA Advanced Energetics Program and was performed under the auspices of the U.S. Department of Energy by Lawrence Livermore National Laboratory under Contract DE-AC52-07NA27344.

\section{REFERENCES}

1. H. K. Mao and R. J. Hemley, "Ultrahigh-pressure transitions in solid hydrogen," Rev. Mod. Phys. 66, 671 (1994)

2. I. Tamblyn and S. A. Bonev, "Structure and Phase Boundaries of Compressed Liquid Hydrogen," Phys. Rev. Lett., 065702 (2010)

3. R. Jeanloz, P. M. Celliers, G. W. Collins, J. H. Eggert, K. K. M. Lee, R. S. McWilliams, S. Brygoo, and P. Loubeyre, "Achieving high-density states through shock-wave loading of precompressed samples," Proc. Natl. Acad. Sci. U.S.A. 104, 9172 (2007)

4. M. R. Armstrong, J. C. Crowhurst, S. Bastea, and J. M. Zaug, "Ultrafast observation of shocked states in a precompressed material," J. Appl. Phys. 108, $023511(2010)$

5. C. A. Bolme, S. D. McGrane, D. S. Moore, and D. J. Funk, "Single shot measurements of laser driven shock waves using ultrafast dynamics ellipsometry," 102, 033513 (2007)

6. J. N. Fritz, "Overtaking wave interaction, reflected shock or reflected release?", AIP Conf. Proc. 370, 271 (1996) 